\title{
Research on Planning of PV-integrated Transmission Grid Considering Operation Efficiency
}

\author{
Zifa Liu ${ }^{1}$, Zhidong Wang ${ }^{2}$, Hanxiao $\mathrm{Yu}^{1,{ }^{1}}$, Xiaoyu Cai ${ }^{1}$, Zhe Zhang ${ }^{1}$ and Jiaming Wang ${ }^{2}$ \\ ${ }^{1}$ School of Electrical and Electronic Engineering, North China Electric Power University, Beijing, China \\ ${ }^{2}$ State Grid Economic and Technological Research Institute CO.LTD, Beijing, China \\ ${ }^{*}$ Corresponding author
}

\begin{abstract}
With the large-scale application of solar in power system, traditional transmission planning of merely focusing on construction investment and operation cost could not meet the practical demands. Based on the evaluation index of the line operation efficiency and the distribution of the power flow, a method for evaluating the operation efficiency of power grid based on the betweenness of active power flow is proposed. On this basis, a transmission grid planning model considering operation efficiency and photovoltaic(PV) curtailment loss is established. The optimization problem is then solved via modified firefly algorithm(MFA) and verified on an IEEE 18-node system. The case study verifies the rationality and validity of the planning model and the method.
\end{abstract}

Keywords_transmission grid planning; PV curtailment loss; operation efficiency

\section{INTRODUCTION}

The development of the power grid evaluation system has been comprehensive evaluation from the single evaluation, and evaluation object developed from current grid state to planning grid. In the early stage, power grid is evaluated mainly by the economic [1], reliability [2-4], adaptability [5], power quality $[6,7]$. In the power grid planning comprehensive evaluation, researchers often focused on the evaluation index system, the quantitative evaluation index model and the comprehensive evaluation method.

At present, there are few methods and standards in operation efficiency evaluation of transmission network equipment, and researches are also carried out less in this field. In [8], an evaluation model based on principal component analysis was proposed including economic efficiency, reliability, safety, stability, equipment utilization level and power quality. The author of [9] proposed a comprehensive operation efficiency evaluation method and established fuzzy neural network evaluation model which combines the intuitive thinking mode and the evaluation experience.

In [10-11], the concept of "betweenness" is introduced to reflect the importance of the node or line, but it does not consider the line transmission capacity or the active power flow factor, so it can not reflect the key degree of the transmission line. In [12-13], the concept of power flow betweenness is proposed and applied to the identification of power system critical lines. The author of [14] established active power flow mediation index, giving weight to different transmission lines and identifying the key lines by weight size.
In grid planning for PV incorporation, [15] comprehensively considers the randomness and regularity of PV output, and presents a model of PV supply probability, which takes into account short-time fluctuation and medium and long-time variation of solar resource. PV curtailment is a problem of large-scale PV integration. [16] analyzes the PV characteristic with energy storage system(ESS) and presents a method of capacity allocation for ESS in to reduce PV curtailment. Considering demand side response, a coordinated planning method is proposed for power grid planning and investment [17].

The Firefly algorithm (FA) is a swarm intelligence algorithm with simple concept and easy implementation [18]. FA can divide solution process into 2 stages: search and adjustment, to improve the convergence speed, so it is suitable for solving the planning model with multiple variables [19]. To avoid premature stagnation of the basic FA, some modification is introduced into iteration process, among them the introduction of mixed chaotic motion is a commonly used improvement [20].

This paper is focused on a transmission grid planning method considering operation efficiency and PV incorporation. The transmission lines are divided into main grid lines and power supply lines according to their functions. Operation efficiency calculation indices are respectively given. Based on active power betweenness and node phase, a grid efficiency evaluating method is proposed. Moreover, on the basis of the traditional planning model, the PV curtailment and operation efficiency is the focus of planning objective. Combined with graph repair strategy, a modified firefly algorithm (MFA) is proposed to solve the planning model.

\section{MOdEL AND Method OF POWER GRID OPERATION EFFICIENCY EVALUATION}

Affected by factors of environment, grid structure and other aspects. Different power grid has different operation efficiency. According to the structure of the power network and functions of the lines, transmission lines can be divided into power supply line and the main line.

The transmission lines capacity can be divided into 2 aspects: (1) economic transmission capacity, the ratio of maximum annual transmission electricity and economic transmission power, reflecting efficiency of transmission lines. (2) stability control limit, the ratio of maximum transmission 
capability and line stability control limit, reflecting efficiency of lines connected generators.

\section{A. Load Rate Model Under Rated Current Carrying Capacity}

Rated current carrying capacity of lines refer to current value with a restricted voltage loss under a determined temperature. This index is determined by material and arrangement of wires and length of the transmission line. The main frame lines mainly undertake the function of power transmission and security. Therefore, the calculation of load factor should refer to economic transmission capacity. The load factor of main frame can be calculated as:

$$
\Phi_{\mathrm{m}}=\frac{P_{\mathrm{m}}}{P_{\mathrm{e}}} \times 100 \%
$$

Where, $\Phi_{\mathrm{m}}$ is load factor of rated carrying capacity for main frame, $P_{\mathrm{m}}$ is maximum transmission capability of lines, $P_{\mathrm{e}}$ is economy transmission capability of lines.

Economy transmission power reflects optimum comprehensive benefits on grid investment, power loss, operation and maintenance costs. If the value is large, the current carrying capacity of the lines performs well.

\section{B. Model of Load Factor on Thermal Stability Limit}

From the perspective of economics, economic transmission power is a optimum and ideal value. Actually, due to the factors of structure and environment, the practical ultimate transmission capacity generally fails to reach the economic transmission capacity. The transmission lines connected generators is relatively independent. Transmission section, i.e trans-provincial transmission channel consisting of two or more transmission lines, is usually scheduled and controlled globally in the whole area. Therefore, referring to stability control limit the load rate factor can be calculated as:

$$
\Phi_{\mathrm{w}}=\frac{P_{\mathrm{m}}}{P_{\mathrm{w}}} \times 100 \%
$$

Where, $\Phi_{\mathrm{w}}$ is load factor of rated carrying capacity for power transmission lines, $P_{m}$ is maximum transmission capability of lines, $P_{\mathrm{w}}$ is stable power control limit of power transmission lines.

\section{Model of Load Factor on Thermal Stability Limit}

In the power grid, the concept of "Betweenness" is introduced to show the importance of nodes or transmission lines [10-11]. However, the capacity of the transmission line or the active power flow factor is not considered, which can't fully and accurately reflect the critical lines in the power grid.

Based on the idea of power flow betweenness, we could identify the critical lines in power grid with weight [12]. The active power transmission is closely related to the phase of the node voltage. Therefore, the active power transmission and the node voltage phase should be considered comprehensively in electrical betweenness index.

Power flow betweenness of lines between node $i$ and node $j$ can be calculated as following:

$$
\begin{gathered}
F_{i j}=k_{i j} \beta_{i j} \\
\beta_{i j}=\sum_{m \in G} \sum_{n \in L}\left|\min \left(P_{m}, P_{n}\right) \cos \theta_{i j}(m, n)\right| \\
k_{i j}=\frac{P_{i j}}{P_{i j, \max }}
\end{gathered}
$$

Where $\min \left(P_{m}, P_{\mathrm{n}}\right)$ is the minimum of active power between generator node $m$ and load node $n, P_{i j}$ is active power transmitted by lines between node $i$ and node $j, P_{i j \max }$ is the maximum active power transmitted by lines between node $i$ and node $j, \theta_{i j}$ is phase difference between node $i$ and node $j, k_{i j}$ is power coefficient, $\beta_{i j}$ is electrical betweenness index considering active power factors. $G$ and $L$ correspondingly represent the generator set and load node set.

Power flow betweenness reveals utilization of transmission lines under current operation condition. The value of power flow betweenness reflects importance of lines[13]. The weights of different lines in the system are represented as follows:

$$
\omega_{i j}=\frac{F_{i j}}{\sum_{\substack{i, j \in N_{1} \\ i \neq j}} F_{i j}}
$$

Where $N_{1}$ is set all branch nodes, $\omega_{i j}$ is weight of branch between node $i$ and node $j$.

\section{OPTIMIZED PLANNING OF TRANSMISSION GRID}

This paper combines the transmission efficiency of power lines with the cost of investment in systems, thus deciding the expansion strategy which takes both operating efficiency and economy into consideration. The objective function of power transmission grid model based on typical scenes mainly includes the operation efficiency, the investment in newly built power lines, the cost of network loss and PV Curtailment loss. 


\section{A. Objective Function}

There is no direct comparison between operation efficiency and operation cost. This paper will put forward a model considering investment cost, the cost of network loss and the loss of PV curtailment which are converted into operation efficiency. It can be generally represented as follow:

$$
\operatorname{minF}=\frac{C_{\mathrm{in}}+C_{\mathrm{op}}+C_{\mathrm{pc}}}{\eta}
$$

Where $F$ is objective function, $\eta$ is power grid operation efficiency, $C_{\mathrm{in}}$ is investment costs of transmission lines, $C_{\mathrm{op}}$ is operation costs, $C_{\mathrm{pc}}$ is loss of PV curtailment.

\section{1) Operation efficiency of power grid:}

According to Section II. A\&B, the operation efficiency of each transmission line can be calculated, then the weight of every line can be got by the method presented in Section II. C, the whole system efficiency is obtained as follow:

$$
\eta=\sum_{\substack{i, j \in N_{\mathrm{GB}} \\ i \neq j}} \omega_{i j} \eta_{i j}+\sum_{\substack{i, j \in\left(N_{\mathrm{B}}-N_{\mathrm{GB}}\right) \\ i \neq j}} \omega_{i j} \eta_{i j}=\sum_{\substack{i, j \in N_{\mathrm{GB}} \\ i \neq j}} \omega_{i j} \frac{\left|P_{i j}\right|}{P_{\mathrm{w}}}+\sum_{\substack{i, j \in\left(N_{\mathrm{B}}-N_{\mathrm{GB}}\right) \\ i \neq j}} \omega_{i j} \frac{\left|P_{i j}\right|}{P_{\mathrm{e}}}
$$

Where $\eta_{i j}$ is operation efficiency of line, $\omega_{i j}$ is weighting of transmission line, $N_{\mathrm{B}}$ is ensemble of all nodes, $N_{\mathrm{GB}}$ is ensemble of all generator nodes, $P_{i j}$ is active power transferred in lines.

\section{2) Capitalized cost of transmission line:}

The investment cost of the new line can be represented as $\mathrm{C}_{\mathrm{in}}$, it can be generally represented as follow:

$$
C_{\text {in }}=\frac{r_{0}\left(1+r_{0}\right)^{m}}{\left(1+r_{0}\right)^{m}-1} \sum_{\substack{i, j \in N_{\mathrm{B}} \\ i \neq j}} n_{i j} c_{i j} L_{i j} Z_{i j}
$$

Where $m$ is service life, $r_{0}$ is discount rate, $n_{i j}$ is new line number between node $i$ and node $j, c_{i j}$ is unit length investment of lines, $L_{i j}$ is length of lines, $Z_{i j}$ represents line construction.

3) Running cost of transmission line:

The operating cost, especially operating loss of lines expressed in $C_{\mathrm{op}}$, is the cost of the equipment in the use of the life cycle of the process. It can be generally represented as follow:

$$
C_{\text {op }}=\sum_{s=1}^{n} P_{\text {loss }} c_{\text {price }} \Delta t_{\text {loss }, s}
$$

Where $c_{\text {price }}$ is power price, $\Delta t_{\text {lossi }}$ is loss hours of typical scenes, $P_{\text {loss }}$ is active power loss.

Active power loss can be calculated as follow:

$$
P_{\mathrm{loss}}=\sum_{\substack{i, j \in N_{\mathrm{B}} \\ i \neq j}} P_{\mathrm{loss}, i j}=\sum_{\substack{i, j \in N_{\mathrm{B}} \\ i \neq j}} \frac{r_{i j} S_{i j}^{2}}{U_{\mathrm{N}}^{2}}
$$

Where $r_{i j}$ is resistance of lines, $S_{i j}$ is transmission power, $U_{\mathrm{N}}$ is rated voltage.

4) Loss of PV curtailment:

Affected by the solar irradiance, the PV output has randomness and the fluctuation, and it's difficult to reach rated output. Loss of PV curtailment can be calculated as follow:

$$
C_{\mathrm{ps}}=c_{\mathrm{pv}} E_{\mathrm{PVC}}= \begin{cases}\frac{r_{0}\left(1+r_{0}\right)^{m}}{\left(1+r_{0}\right)^{m}-1} c_{\mathrm{pv}} \sum_{i=1}^{n}\left(P_{\mathrm{pv}}(t)-P_{\mathrm{T}}\right) \Delta_{i}, & P_{\mathrm{T}}<P_{p v m a x} \\ 0 & , P_{\mathrm{T}} \geq P_{p v m a x}\end{cases}
$$

Where $c_{\mathrm{pv}}$ is price of $\mathrm{PV}$ power, $E_{\mathrm{PVC}}$ is power loss of $\mathrm{PV}$ curtailment, $P_{p v i(t)}$ is output of PV power curve in scene $i, P_{\mathrm{T}}$ is transmission capacity limit of lines, $\Delta t_{i}$ is duration of $\mathrm{PV}$ curtailment in scene $i$.

\section{B. Constrains}

1) Constrains of power balance:

$$
\left\{\begin{array}{l}
P_{\mathrm{G}, i}-P_{\mathrm{L}, i}=U_{i} \sum_{j=1}^{N_{\mathrm{B}}} U_{j}\left(G_{i j} \cos \theta_{i j}+B_{i j} \sin \theta_{i j}\right) \\
Q_{\mathrm{G}, i}-Q_{\mathrm{L}, i}=U_{i} \sum_{j=1}^{N_{\mathrm{B}}} U_{j}\left(B_{i j} \cos \theta_{i j}-G_{i j} \sin \theta_{i j}\right)
\end{array}\right.
$$

Where $P_{\mathrm{G}, i}, Q_{\mathrm{G}, i}$ represent output of generator, $P_{\mathrm{L}, i}, Q_{\mathrm{L}, i}$ represent active power and reactive power of load. $U_{i}$ is node voltage, $G_{i j}, B_{i j}$ respectively represent conductance, susceptance.

2) Constrains of node voltage:

$$
U_{i, \min } \leq U_{i} \leq U_{i, \max }
$$

Where $U_{i, \min }, U_{i, \max }$ are voltage limit.

3) Constrains of output power of generating set:

$$
P_{G, i, \min } \leq P_{G, i} \leq P_{G, i, \max }
$$

Where $P_{G i, \min }, P_{G i, \max }$ are output power limit of generator at node $i$.

4) Constrains of transmitting power of power grid:

$$
P_{i j} \leq P_{i j, \max }
$$


Where $P_{i j}$ represent active power between node $i$ and node $j$, $P_{i j \text { max }}$ is maximum transmission power.

\section{MODIFIED FIREFLY ALGORITHM(MFA)}

The Firefly algorithm (FA) is a random optimization algorithm based on the swarm intelligence. Firefly algorithm has the characteristics of high computational precision and fast convergence speed, and has been applied to image processing [21], data mining [22] and power system [23]. This paper introduces graph theory to repair of unconnected particles, so the global optimization ability is efficiently improved. In the FA algorithm, the direction and distance of the Firefly is mainly determined by the brightness and the degree of attraction, the fluorescence degree determines the target function value, and the fluorescence brightness determines the position of the Firefly. At the initial moment, all fireflies are randomly distributed in space.

\section{A. Parameter Variance Adjustment Strategy}

The FA algorithm has the disadvantage of poor initial search precision and easy to fall into the local optimum in late search. Some work has studied the impact of step factor $\alpha$, attraction factor $\zeta$ and optical absorption coefficient $\gamma$ on precision and convergence speed. A modified FA on parameter variance regulation is proposed in this work. The core idea of this improvement to use different parameter structures in 2 stages. The population divergence is defined as the initial iterative process, and the population convergence is defined as the later iterative process of optimization. The variation of population divergence was measured by calculating the brightness variance of fireflies.

Normalized operator is defined as (17)

$$
I=\left\{\begin{array}{cc}
\max \left(\left|I_{i}-I_{\text {avg }}\right|\right), & \max \left(\left|I_{i}-I_{\text {avg }}\right|\right)>1 \\
1, & \max \left(\left|I_{i}-I_{\text {avg }}\right|\right) \leq 1
\end{array}\right.
$$

Where, $I_{i}$ is the brightness of firefly particle $i, I$ represents the normalized brightness, $I_{\text {avg }}$ is the average brightness of firefly particles.

Variance of brightness is defined as (18)

$$
\sigma_{I}^{2}=\frac{1}{n_{\mathrm{F}}} \sum_{i=1}^{n_{\mathrm{F}}}\left(\frac{I_{i}-I_{\mathrm{avg}}}{I}\right)^{2}
$$

Where, $n_{\mathrm{F}}$ is the number of firefly particles.

Step factor $\alpha$, attraction factor $\zeta$ and optical absorption coefficient $\gamma$ update as (19)

$$
\left\{\begin{array}{l}
\alpha_{i}=\alpha_{b}+e^{-k_{\mathrm{F}} \sigma_{I}^{2}}\left(\alpha_{e}-\alpha_{b}\right) \\
\gamma_{i}=\gamma_{b}+e^{-k_{\mathrm{F}} \sigma_{I}^{2}}\left(\gamma_{e}-\gamma_{b}\right) \\
\zeta_{i j}(t)=\zeta_{0} J(t) e^{-\gamma(t) r_{i j}^{2}}
\end{array}\right.
$$

Where, subscript $b$ represents beginning, $e$ represents ending, $k_{\mathrm{F}}$ is adjustment coefficient. The variance $\sigma_{I}$ is used to reflect the degree of convergence of the firefly population.

The corresponding location update formula can be expressed as (20)

$$
X(i+1)=X(i)+K \zeta_{i j}(X(j)-X(i))+\alpha(\text { rand }-0.5)
$$

$$
K=\operatorname{Light}(j) / \operatorname{Light}(i)
$$

In this case, the artificial introduction of parameter $K$ to improve the attraction of fireflies can help to find a better solution quickly.

\section{B. Graph Repair Strategy}

In this paper, a particle repair method is used to reduce the generation of the failure particles and improve the operation speed [24].

Steps are taken as follows:

(1) Find out the important node or node set represented in the subgraph particle as isolated nodes;

(2) Search out and verify all the connected piece, if connected, turn to step (4), otherwise to step (3);

(3) Process each independent connected region as a node, verify the existence of minimum spanning tree, if exist, turn to step (5), else turn to step (6);

(4) Using Boruvka algorithm for minimum spanning tree, turn to step (6);

(5) Find and intersect the node set represented by each node in the original graph in each connected region. If the corresponding connected region node is not contained in the intersection set, any node of the intersection set is added to the sub graph, otherwise, the node in a non sub graph is taken into account, then turn to step (3);

(6)The corresponding decision variable is set to 1 in the additional branch.

Flow chart of MFA can be represented as Figure I. 


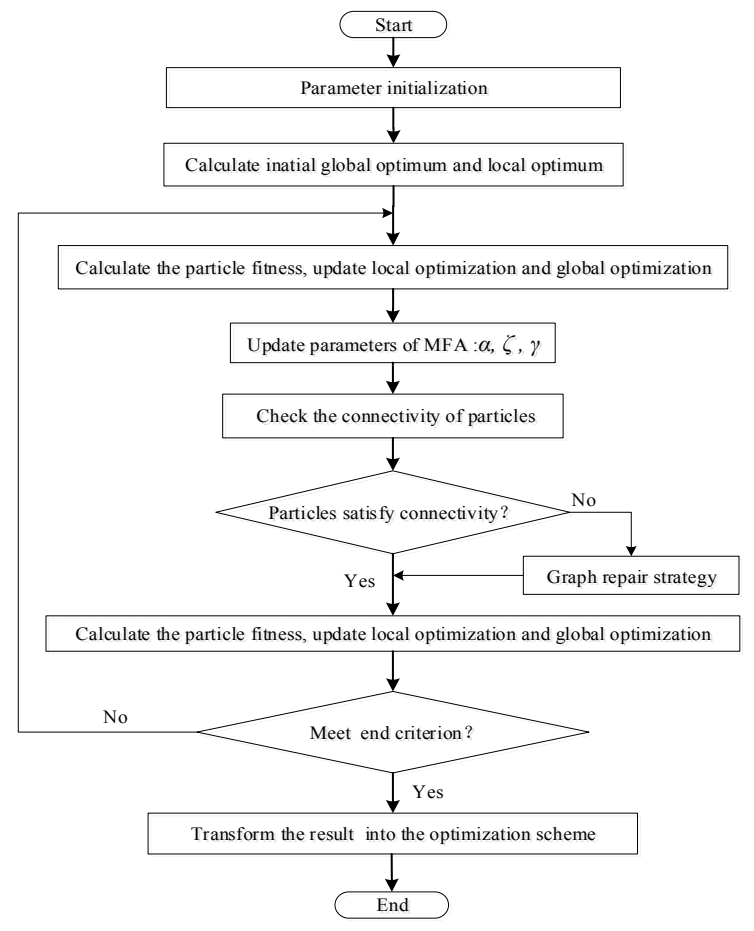

FIGURE I. FLOW CHART OF MFA

\section{CASE STUDY}

In this paper, an improved IEEE18 node system is used for case study. Topology as shown in the Figure II. The existing lines are represented by 9 solid lines, 35 dotted lines indicate the route to be selected. In node 14, PV installed capacity is $600 \mathrm{MW}$. The new line is $10^{6} ¥ / \mathrm{km}$. The voltage reference value is $220 \mathrm{kV}$, the PV price is $0.56 ¥ / \mathrm{kW} \cdot \mathrm{h}$, the curtailment loss of $\mathrm{PV}$ is $0.65 ¥ / \mathrm{kW} \cdot \mathrm{h}$. The planning period is 10 years.

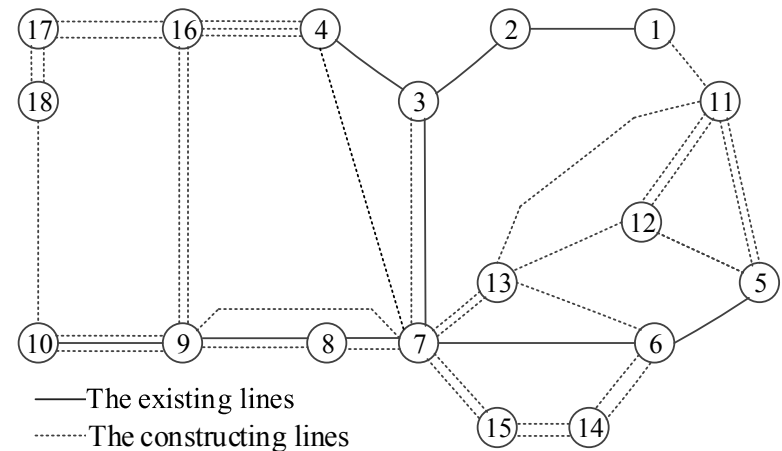

\section{FIGURE II. STRUCTURE DIAGRAM OF 18 NODE SYSTEM}

Because of the uncertainty and volatility of PV output, it is impossible to simply select the output data of a certain day or a certain period of time. On the basis of 3 years' photovoltaic continuous generation and large load data in Qinghai area, feature extraction and k-mean scene clustering analysis are carried out, and four typical scenes are obtained. The probability ratio of Scene 1 to scene 4 is $0.17: 0.45: 0.13: 0.25$, the maximum active power output (unitary value) and the average load size (unitary value) of each scene are shown as Table I.

\section{TABLE I. MAXIMUM OUTPUT OF PV AND AVERAGE LOAD CAPACITY IN TYPICAL SCENES}

\begin{tabular}{|c|c|c|}
\hline Typical scene & $\begin{array}{c}\text { PV output } \\
\text { (unitary value) }\end{array}$ & $\begin{array}{c}\text { Average load } \\
\text { size (unitary } \\
\text { value) }\end{array}$ \\
\hline Scene 1 & 0.6 & 0.8 \\
\hline Scene 2 & 0.5 & 1.1 \\
\hline Scene 3 & 0.8 & 0.7 \\
\hline Scene 4 & 0.7 & 0.9 \\
\hline
\end{tabular}

According to the MFA and debugging experience, the population number $\mathrm{z}=20$ and maximum iteration number $\mathrm{k}=100$ are set. According to the data of four typical scenarios, the planning plan (planning scheme 1) is obtained as shown in Figure III. Under this plan, the load rate, operation efficiency and investment data of each scenario are shown in Table II and Table III. According to the probability of various scenarios and the data in Table III, we can get various indicators of the planning plan, as shown in Table IV.

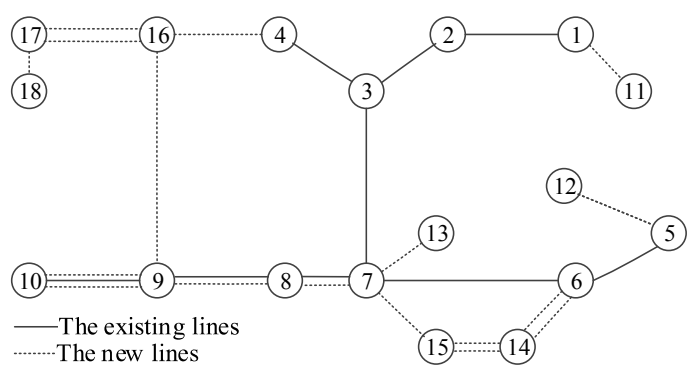

FIGURE III. PLANNING SCHEME 1

TABLE II. LOAD RATE OF LINES IN FOUR TYPICAL SCENES

\begin{tabular}{|c|c|c|c|c|}
\hline Lines & $\begin{array}{c}\text { Load rate } \\
\text { (scene 1)/\% }\end{array}$ & $\begin{array}{c}\text { Load rate } \\
\text { (scene 2)/\% }\end{array}$ & $\begin{array}{c}\text { Load rate } \\
\text { (scene 3)/\% }\end{array}$ & $\begin{array}{c}\text { Load rate } \\
\text { (scene 4)/\% }\end{array}$ \\
\hline $1-2$ & 77.23 & 83.26 & 69.21 & 53.35 \\
\hline $1-11$ & 72.71 & 77.22 & 71.83 & 66.87 \\
\hline $2-3$ & 58.67 & 64.41 & 59.24 & 62.23 \\
\hline $3-4$ & 57.22 & 59.21 & 51.79 & 61.18 \\
\hline $3-7$ & 63.93 & 70.35 & 63.40 & 57.29 \\
\hline $4-16$ & 54.64 & 63.72 & 47.27 & 67.14 \\
\hline $5-6$ & 64.59 & 71.70 & 68.63 & 46.21 \\
\hline $5-12$ & 56.32 & 61.32 & 55.24 & 73.52 \\
\hline $6-7$ & 62.23 & 68.81 & 63.62 & 48.13 \\
\hline $6-14$ & 41.41 & 47.27 & 46.46 & 69.36 \\
\hline $7-8$ & 73.08 & 78.64 & 67.47 & 59.28 \\
\hline $7-13$ & 41.85 & 48.69 & 41.12 & 62.81 \\
\hline $7-15$ & 65.23 & 71.39 & 61.11 & 51.20 \\
\hline $8-9$ & 47.17 & 59.70 & 58.29 & 67.47 \\
\hline $9-10$ & 57.24 & 64.21 & 58.23 & 71.18 \\
\hline $9-16$ & 49.50 & 53.63 & 46.07 & 64.32 \\
\hline $14-15$ & 62.22 & 67.71 & 62.19 & 53.31 \\
\hline $16-17$ & 68.47 & 75.13 & 56.79 & 66.93 \\
\hline $17-18$ & 63.49 & 68.28 & 61.03 & 62.49 \\
\hline
\end{tabular}


TABLE III. OPERATION EFFICIENCY AND COSTS IN FOUR TYPICAL SCENES

\begin{tabular}{|c|c|c|c|c|}
\hline scenes & $\begin{array}{c}\text { Line cost/ } \\
\quad 10^{6} ¥\end{array}$ & $\begin{array}{c}\text { Loss } \\
\text { Charges/1 } \\
0^{6} ¥\end{array}$ & $\begin{array}{l}\text { curtailme } \\
\text { nt loss of } \\
\text { PV } / \mathbf{1 0}^{6} ¥\end{array}$ & $\begin{array}{c}\text { operating } \\
\text { efficiency } \\
/ \%\end{array}$ \\
\hline Scene 1 & 270.79 & 4.70 & 80.78 & 59.85 \\
\hline Scene 2 & 270.79 & 4.83 & 61.33 & 65.93 \\
\hline Scene 3 & 270.79 & 4.74 & 11.27 & 58.82 \\
\hline Scene 4 & 270.79 & 4.80 & 78.86 & 62.96 \\
\hline
\end{tabular}

TABLE IV. EACH INDEX IN PLANNING SCHEME

\begin{tabular}{|c|c|c|c|c|}
\hline $\begin{array}{c}\text { Comprehensiv } \\
\text { e investment } \\
\text { of lines } / \mathbf{1 0}^{\mathbf{6}} \mathbf{¥}\end{array}$ & $\begin{array}{c}\text { curtailm } \\
\text { ent loss } \\
\text { of } \\
\mathbf{P V} / \mathbf{1 0}^{\mathbf{6}} \mathbf{¥}\end{array}$ & $\begin{array}{c}\text { Operating } \\
\text { efficiency/ } \\
\mathbf{\%}\end{array}$ & $\begin{array}{c}\text { Total } \\
\text { investment } \\
\mathbf{1 0}^{\mathbf{6}} \mathbf{\Xi}\end{array}$ & $\begin{array}{c}\text { objective } \\
\text { function }\end{array}$ \\
\hline 289.95 & 75.70 & 63.23 & 365.65 & 578.29 \\
\hline
\end{tabular}

The objective function of this paper is to consider the investment cost of the power grid construction, the operating cost of the system, the curtailment loss of PV and the operation efficiency of the power grid, while the traditional power grid planning focuses on the economics of planning the grid structure, that is, the minimum investment in line construction and the minimum network loss as the objective function.

$$
\min \mathrm{F}=C_{\text {in }}+C_{\text {op }}
$$

Solving the above planning model, the plan (planning scheme 2) obtained is shown in Figure IV, and the comparison of the two schemes is shown in Table V.

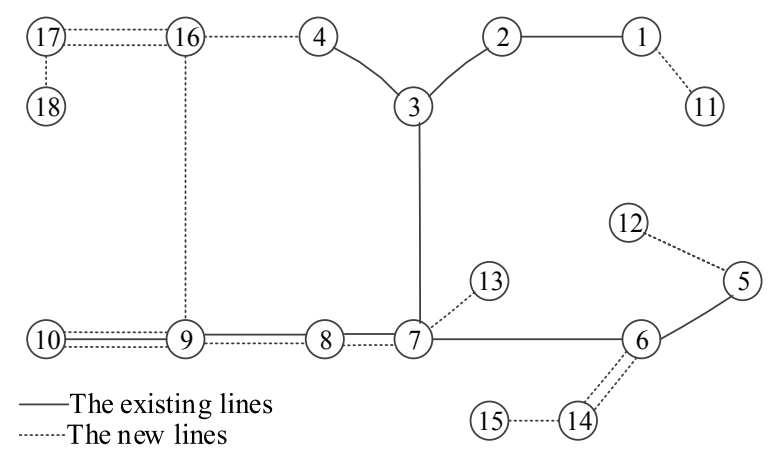

FIGURE IV. PLANNING SCHEME 2

Comparing the extension lines of the two schemes, we can see the branch road 14-15 needs to build a double line and increases the branch $7-15$ in the scheme 1 , while there are no these lines in the scheme 2. It means that the one-time investment of scheme 2 is a little lower than the scheme proposed in this paper. However, curtailment loss of PV in scheme 2 is much higher than scheme 1 . The reason is that scheme 1 increases the send channels of PV power. Although the operation efficiency is reduced, maximum transmission capacity of transmission lines is increased and the blocking effect of photovoltaic transmission lines is alleviated. The objective function of scheme 2 only considers line investment and operation loss cost, therefore, the scheme 2 is the most economical plan which ensures the safety of the system. Focusing on the Table V, we can see that the load rate of most lines in scheme 2 is higher and the overall efficiency of power grid is $1.75 \%$ higher than that of scheme 1 . From the view of $\mathrm{PV}$ consumption, the PV curtailment rate of scheme 2 is $4.82 \%$ higher than that of plan 1 , meaning that the curtailment loss of PV in scheme 2 is much larger. The total investment in planning years is $8.25 \%$ higher than that of scheme 1 . It can be seen that the scheme of the power grid planning model proposed in this paper can greatly reduce the curtailment loss of PV under the premise of guaranteeing the operation efficiency.

TABLE V. COMPARISON OF TWO PLANNING SCHEMES

\begin{tabular}{|c|c|c|}
\hline Index & Planning scheme 1 & Planning scheme 2 \\
\hline $\begin{array}{l}\text { Optimize Grid } \\
\text { Information } \\
\text { (number of } \\
\text { extension lines) }\end{array}$ & \begin{tabular}{|c|}
$1-11(1), 4-16(1), 5-$ \\
$12(1), 6-14(2), 7-8(1)$ \\
$8-9(1), 9-10(2), 9-$ \\
$16(1), 7-13(1), 7-15(1)$ \\
$14-15(2), 16-17(2), 17-$ \\
$18(1)$
\end{tabular} & $\begin{array}{c}1-11(1), 4-16(1), 5- \\
12(1), 6-14(2), 7-8(1), \\
8-9(1), 9-10(2), 9- \\
16(1), 7-13(1), 14- \\
15(1), 16-17(2), 17- \\
18(1)\end{array}$ \\
\hline $\begin{array}{c}\text { Comprehensive } \\
\text { investment of } \\
\text { lines } / 10^{6} ¥\end{array}$ & 289.95 & 278.96 \\
\hline $\begin{array}{c}\text { Rate of PV } \\
\text { curtailment } / \%\end{array}$ & 9.68 & 14.50 \\
\hline $\begin{array}{l}\text { Curtailment loss } \\
\text { of PV } / 10^{6} ¥\end{array}$ & 75.70 & 116.84 \\
\hline $\begin{array}{c}\text { Operation } \\
\text { efficiency of PV } \\
\text { transmission line }\end{array}$ & \begin{tabular}{|c|} 
Operation efficiency of \\
$6-14$ is $51.72 \%$ \\
Operation efficiency of \\
$14-15$ is $62.45 \%$ \\
\end{tabular} & $\begin{array}{c}\text { Operation efficiency } \\
\text { of } 6-14 \text { is } 51.74 \% \\
\text { Operation efficiency } \\
\text { of } 14-15 \text { is } 63.08 \% \\
\end{array}$ \\
\hline $\begin{array}{c}\text { Operation } \\
\text { efficiency of } \\
\text { Power grid } / \% \\
\end{array}$ & 63.23 & 64.98 \\
\hline $\begin{array}{c}\text { Total investment } \\
/ 10^{6} ¥\end{array}$ & 365.65 & 395.80 \\
\hline $\begin{array}{l}\text { Objective } \\
\text { function }\end{array}$ & 578.29 & 609.11 \\
\hline
\end{tabular}

In summary, the power transmission network planning model proposed in this paper which considering the operational efficiency can effectively choose the more reasonable power grid planning scheme, which balances the operation efficiency and the economy of the power grid. Meanwhile, the serious problem of PV curtailment loss caused by the PV power connection can be alleviated. This model may have more practical significance.

\section{CONCLUSION}

In this paper, the operational efficiency factors are taken into account in the power grid planning model based on the evaluation index and method of line operation efficiency. A method based on active power flow betweenness is proposed, which gives different weights according to the importance of different lines in the power grid, which can give the contribution of different lines to the operation efficiency of power grid according to the phase of different nodes and active power flow distribution in the power grid. In the transmission network planning model, the objective function considers the cost of investment, operating cost, operation efficiency and PV curtailment loss. Aiming at the shortcomings of FA, the MFA 
is proposed in this paper to solve the planning model, which changing the parameters to avoid falling into local optimum and improving the speed of the algorithm through graph theoretic repair strategy. The case study is compared and analyzed whether the objective function takes into account the impact of operation efficiency and PV curtailment loss on the power grid planning, showing that the planning model proposed in this paper can take into account the economic and operational efficiency of the power grid planning, and providing a solution for the transmission network planning considering the curtailment loss of PV and the efficiency of the power grid.

\section{REFERENCES}

[1] W. H. Tang, K. Spurgeon, Q. H. Wu, Z. J. Richardson. An Evidential Reasoning Approach to Transformer Condition Assessments. IEEE Trans on Power Delivery. 2004, 19(4), 1696-1703.

[2] Qian Z, Yan Z. Fuzzy Synthetic Method for Life Assessment of Power Transformer. IEEE Proceedings: Science and Measurement Technology. 2004, 151(3): 175-180

[3] Qu Gang, Cheng Haozhong, MA Zeliang, Zhu Zhonglie, Zhang Jianping, Yao Zhongliang. Multi-objective Transmission Expansion Planning Considering Generation Capacity Adaptability. Automation of Electric Power System.2009, 33(23): 19-23.

[4] Yuan Xiaodong, Zhao Jianfeng, TANG Guoqing, Han Zhengzhong. Multi-level Fuzzy Comprehensive Evaluation of Power Quality. International Conference on Electric Utility Deregulation, Restructuring and Power Technologies, Hong Kong. 2004, 4: 290-294

[5] Liu Sige, Cheng Haozhong, Cui Wenjia. Optimal Model of Multiobjective Electric Power Network Planning Based on Rough Set Theory. Proceedings of the CSEE. 2007, 27(7): 65-69.

[6] Tang Huizhi, Peng jianchun. Research on Synthetic and Quantificated Appraisal Index of Power. Power System Technology. 2003, 27(12): 8588.

[7] Romero R, Monticelli A, Garcia A, Haffner S. Test Systems and Mathematical Models for Transmission Network Expansion Planning. IEEE Proceedings: Generation, Transmission and Distribution. 2002, 149(1): 27-36.

[8] Song Lingli, Yang Jun. A Method of Full-Scale Comprehensive Evaluation on Operating Efficiency of Electric Power Networks. Journal of Electric Power. 2015, 03: 268-274.

[9] Song Lingli, Jia Legang, Zhou Bowen. Research on Operational Efficiency Assessment of Power Grid Based on Fuzzy Neural Network. HEBEI ELECTRIC POWER. 2015, 03: 30-32+54.

[10] Motter A E, Lai Y-C. Cascade-based attacks on complex networks. Physical Review E. 2002, 66(2): 1-4.

[11] Kinney R, Crucitti P, Albert R, Latora V. Modeling cascading failures in the North American power grid[J]. European Physical Journal B. 2005, 46(1): 101-107.

[12] Liu Wenying, Liang Cai, Xu Peng, Dan Yangqing, Wang Jiaming, Wang Weizhou. Identification of Critical Line in Power Systems Based on Flow Betweenness. Proceedings of the CSEE. 2013, 31: 90-98+11.

[13] Liang Cai, Liu Wenying, Dan Yangqing, Xu Peng, Zhou Xichao. Flow Betweenness of Transmission Lines and Its Application in Critical Line. Automation of Electric Power System. 2014, 08:35-40.

[14] Zhang Tao, Sun Xiaowei, Xu Xueqin, Li Zhenxing. Identification of Critical Lines in Power Grid Based on Active Power Flow Betweenness. Power System Technology. 2016, 01:193-198.

[15] Guan Lin, Chen Xu, Lv Yaotang, Tang Zongshun, Zhao Qi.Probability model of PV generation for power system planning and its application[J]. Electric Power Automation Equipment, 2017, 37(11):1-7.

[16] Gong Yu, Li Na, Liu Ximei, et al. Configuration Method for Capacity of Energy Storage in PV Station Considering PV Power Curtailment. NORTH CHINA ELECTRIC POWER, 2017(10): 33-37.
[17] Armendáriz M, Heleno M, Cardoso G, Mashayekh S, Stadler M, Nordström L. Coordinated microgrid investment and planning process considering the system operator. Applied Energy, 2017, 200:132-140.

[18] Fang Biwu, Wang Bo, Liu Dichen, Luo Jinhao, Ma Hengrui, Chen Siyuan. A two-stage firefly algorithm based on search + adjustment for solving unit commitment problem. Power System Protection and Control, 2016, 44(23): 17-23.

[19] Liu Wenxue, Liang Jun, Yun Zhihao, Li Zhengmao, Dong Xiaoming. Multi-objective Fuzzy Chance Constrained Dynamic Economic Dispatch Considering Energy Saving and Emission Reduction. TRANSACTIONS OF CHINA ELECTRICAL SOCIETY, 2016, 31(1): 62-70

[20] Wang Xin, Huang Ke, Zheng Yihui, Shao Fengpeng, Jia Likai, Xu Qingshan. Combined PV Power Forecast Based on Firefly AlgorithmGeneralized Regression Neural Network. Power System Technology, 2017, 41(2): 455-461.

[21] Zhao Kai, Han Yi, Luo Shengyong. The Image Retrieval Based on Attribute Feature Extraction Coupled Firefly Optimization. MICROELECTRONICS \& COMPUTER, 2017(11): 31-36[23]

[22] Luo Dongsong, Li Xiongwei, Zhao Xiaoqiang. Research on fuzzy clustering algorithm based on GSO. Industrial Instrumentation \& Automation, 2013(2): 3-6.

[23] Li Fangjuan, Xue Jianmin, Zhou Xianghua, Yang Kan, Zhu Dawei, Wang Xinkui, Liang Yongjing. Application of Firefly Algorithm in Unit Commitment of Large Hydropower Station. Water Resources and Power 2018, 36(03): 164-167+160.

[24] Bian Zhao, Tang Ping, Chen Chenxin. Region merging method based on threshold constrained minimum spanning tree. Computer Engineering and Design, 2012, (1): 229-232+242. 